\title{
PREPARATION OF OXYMATRINE N-BUTYL POLYCYANOACRYLATE NANOPARTICLES AND ITS HEPATOPROTECTIVE EFFECTS IN OB/OB MICE
}

\author{
JING WU4, PEI YANG ${ }^{2}$, XUEQIN JIN ${ }^{1}$, WEIHUA LI' ${ }^{1}$, SHENGCUN HE ${ }^{1}$, \\ JIANMAO $\mathrm{CHEN}^{1 *}$ and DONGTAO $\mathrm{ZHANG}^{3 *}$
}

'Experimental Animal Center, Ningxia Medical University, Yinchuan 750001, China

${ }^{2}$ Department of Otolaryngology-Head and Neck Surgery. The General Hospital of Ningxia Medical University, Yinchuan 750004, China

${ }^{3}$ College of Life Sciences, Ningxia University, Yinchuan 750001, China

${ }^{4}$ Laboratory Animal Center, Xi'an Jiaokong University Health Science Center, Xi'an, Shaanxi, 710061, China

\begin{abstract}
This study aims to prepare oxymatrine n-butyl polycyanoacrylate nanoparticles (OM-PBCA-NPs) and observe its hepatoprotective effects in ob/ob mice. Using oxymatrine as the drug and n-butyl cyanoacrylate (BCA) as the carrier to prepare OM-PBCA-NPs by the emulsion polymerization method; ob/ob mice were used as the mouse model of liver injury, given OM-PBCA-NPs, and collected the blood at the end of Week 12 of the experiment to detect the blood lipids, serum alanine aminotransferase (ALT), and aspartate aminotransferase (AST). Under SEM, the particles of OM-PBCA-NPs were round and well-formed with no adhesion. The average particle size was $(104.1 \pm 29.11) \mathrm{nm}$, the distribution was narrow, and the particle size met the requirements of particle size for liver targeted therapy. The encapsulation efficiency was $83 \%$ and the drug load amount was 16.6\%. Moreover, OM-PBCA-NPs can significantly reduce the blood lipid level and serum ALT/AST contents in mice with fatty liver injury, and its effects were better than common oxymatrine granules. OM-PBCA-NPs has good liver targeting effects, and its hepatoprotective and lipid-lowering effects are superior to those of common oxymatrine granules.
\end{abstract}

Keywords: oxymatrine n-butyl polycyanoacrylate nanoparticles, OM-PBCA-NPs, oxymatrine, fatty liver injury

Oxymatrine (OMT), also known as oxymatrine, is an alkaloid extracted from Chinese traditional medicine Radix et Rhizoma Sophorae Tonkinesis $(1,2)$ and is clinically used for the treatment of chronic hepatitis (3-5), which can effectively reduce the serum contents of AST and ALT and improve clinical symptoms (6-8); however, common oxymatrine preparations require multiple administration, and the doses are large, which may result in great fluctuation of such patients' blood concentration; they also have greater toxic side effects $(9,10)$. This brings certain difficulties and problems to the clinical application of OMT. It has become one of the hotspots in the current research to improve the new drug structure and mode of action to enable the pharmacological effects of OMT to play an important role in target organs (11-13).

\footnotetext{
* Corresponding author: e-mail: dongtaozhangcn@163.com
}

Solid lipid nanoparticles (SLN) matrix materials are high-melting natural or synthetic solid lipids with physiological compatibility and biodegradability. They are widely concerned as new colloidal drug carriers because they can replace traditional carrier systems (14). In the preparation, suitable lipid materials can be selected according to different physical and chemical properties of the drug and preparation process, also several different types of mixed lipids can be used as carriers (14). The currently used polymer nanoparticle carrier materials are synthetic biodegradable polymer materials, such as polyalkyl cyanoacrylates, methyl methacrylate, and polylactic acid (14). Alkyl cyanoacrylate is mainly used for the preparation of drug-loaded nanoparticles because of its in vivo targeting, drug release continuity, biodegradability, ease of preparation, low toxicity, high drug loading, and auto- 
clavable sterilization (15). It has a very important industrial and clinical application value. At present, the preparation methods of polyalkyl cyanoacrylate nanocarriers mainly include emulsion polymerization, interface condensation and interface deposition (16). Compared with these three preparation methods, the emulsion polymerization method is easy to operate and the conditions are easy to control, which is more suitable for OMT of water-soluble.

In this study, the emulsion polymerization method was used to prepare OM-PBCA-NP, which was used as a targeted drug delivery system. These preparations have the characteristics of targeted transportation, reduced systemic drug distribution, reduced dosage and administration frequency, which can improve drug treatment indicators and reduce adverse reactions in patients.

\section{MATERIALS AND METHODS}

\section{Preparation of oxymatrine nanoparticles Determination of standard curve}

Accurately weighed oxymatrine $(10 \mathrm{mg}$, provided by NIFDC, batch no: 110780-201508) was placed in a $25 \mathrm{~mL}$ volumetric flask, dissolved in distilled water, diluted to the volume, and shaken well to prepare the stock solution; precise amounts of oxymatrine stock solution $(25,50125,250,500$, 1000 , and $2000 \mu \mathrm{L}$ ) were then sampled, placed in 10 $\mathrm{mL}$ volumetric flasks, diluted to the volume with distilled water, and shaken well, respectively. $20 \mu \mathrm{L}$ of each solution was then injected into HPLC, which repeated three times to calculate the average value for preparing the standard curve based on the peak area.

\section{Preparation of OM-PBCA-NP}

1. $50 \mathrm{mg}$ of OMT (17), $100 \mathrm{mg}$ of Dextran D-70 (Beijing Biotopped Technology Co., Ltd.170280-01), $100 \mathrm{mg}$ of Block Polyether F-68 (Shanghai Yuanye Biotechnology Co. Ltd., XJ0621TA13), $50 \mathrm{mg}$ of sodium hydrogen sulfite (Tianjin Damao Chemical Reagent Factory, 20151019), and $0.1 \mathrm{~mL}$ of BCA (Beijing Shunkang Technology Development Co., Ltd. 20160703).

2. The above OMT, D-70, F-68, and sodium hydrogen sulfite were sequentially added into one beaker and sonicated in $10 \mathrm{~mL}$ of distilled water. The $\mathrm{pH}$ was adjusted to 2 with $0.1 \mathrm{~mol} / \mathrm{L}$ hydrochloric acid (Kunxin Jincheng Reagent Co. China).

3. The beaker with OMT was placed on a magnetic stirrer (IKA, Germany, 600 r/min), dropwise added $0.1 \mathrm{ml}$ of BCA, stirred for $2 \mathrm{~h}$, adjusted the $\mathrm{pH}$ to 7 using $0.1 \mathrm{~mol} / \mathrm{L} \mathrm{NaOH}$ (Tianjin Guangfu Technology Development Co. Ltd., 20130202), and then continued stirring for $0.5 \mathrm{~h}$.

4. The solution was filtered through a $0.45 \mu \mathrm{m}$ microporous membrane to the removal of unformed or larger-sized nanoparticles. The continuous filtrate is the OM-PBCA-NP suspension.

\section{Morphological observation}

An appropriate amount of the prepared nanoparticle colloidal solution was taken and dropped on a copper mesh special for transmission electron microscope (H-7650 HITACHI, Japan), negatively stained with $1.5 \%$ phosphotungstic acid, naturally dried, and observed by TEM.

\section{Particle size and distribution}

The OM-PBCA-NP sample was firstly separated by a High-speed refrigerated centrifuge (Centrifuge5430R, Eppendorf $)\left(4^{\circ} \mathrm{C}, 15000 \mathrm{r} / \mathrm{min}, 150\right.$ min), and observed the morphology and photographed.

\section{Determination of encapsulation efficiency}

The encapsulation efficiency of OM-PBCANP was determined by ultrarapid freezing centrifugation (HITACHI, Japan). $1 \mathrm{~mL}$ of the colloidal solution was taken and placed into one centrifuge tube, followed by freezing centrifugation $\left(4^{\circ} \mathrm{C}\right.$, $40000 \mathrm{r} / \mathrm{min}, 120 \mathrm{~min}$ ), the $0.5 \mathrm{~mL}$ supernatant of which was then diluted, metered to the volume, and determined the concentration by HPLC (Agilent1260, American) referring to the calculation formula of nanoparticle encapsulation ratio issued in "Chinese Pharmacopoeia" 2005 edition:

Encapsulation efficiency $(\%)=\left(\mathrm{W}_{\text {total }}-\mathrm{W}_{\text {free }}\right) / \mathrm{W}_{\text {tota }} \times$ $100 \%=\left(\mathrm{A}_{0}-\mathrm{A}_{1}\right) / \mathrm{A}_{0} \times 100 \% . \mathrm{W}_{\text {total }}$ : the total amount of drug; $\mathrm{W}_{\text {free }}$ : the amount of free drug

$\mathrm{A}_{0}$ : Peak area of total drugs; $\mathrm{A}_{1}$ : Peak area of free drugs

\section{Determination of drug loading}

$\mathrm{DL}=\mathrm{W}_{\mathrm{E}} / \mathrm{W}_{\text {total }} \times 100 \%$

DL: drug loading; $\mathrm{W}_{\mathrm{E}}$ : Amount of drug encapsulated in nanoparticle; $\mathrm{W}_{\text {total }}$ : Total dosage of nanoparticle.

\section{Stability test}

OM-PBCA-NPs colloidal solution was placed at room temperature $\left(20-30^{\circ} \mathrm{C}\right)$ for 3 months, the morphology was observed, the particle size, entrapment efficiency, and drug loading were measured. 


\section{Grouping and treatment}

Ten Specific Pathogen Free (SPF) C57BL/6J mice (Experimental Animal Center, Ningxia Medical University, production license number: SCXK (Ning) 2005-0001); standard bodyweight, $\mathrm{M}: \mathrm{F}=1: 1)$ were set to the normal group $(\mathrm{CON})$; a total of 40 SPF ob/ob mice (Experimental Animal Center, Ningxia Medical University, production license number: SCXK (Ning) 2005-0001; standard body weight (18-22 g), half male and half female $(\mathrm{M}: \mathrm{F}=1: 1$ ) were randomly divided into 4 groups, with ten mice in each group, namely the model group (MOD), the OM-PBCA-NP group (OM, 205 $\mathrm{mg} / \mathrm{Kg}$ ), the OMT group (OMT, $30 \mathrm{mg} / \mathrm{Kg}$ ), and the silymarin group (SIL, $36 \mathrm{mg} / \mathrm{Kg}$, MADAUS,
Germany, B1403403, as the positive control group). The mice in group CON were given normal saline, and the other groups were given the corresponding drugs for 12 weeks. At the end of Week 12, the mice were killed and collected the serum. The blood lipid content and plasma ALT were detected using one automatic biochemical analyzer (SYSMEX JCABM6010/C) (Huaxin Technology Co. Ltd., Guangzhou, AST:170814; AST:170706; TC : 170907; HLDL:381AGC; LDL:379AHG; TG:1810104).

\section{Statistical processing}

The measurement data were processed using SPSS 11.5 for statistical one-way ANOVA and

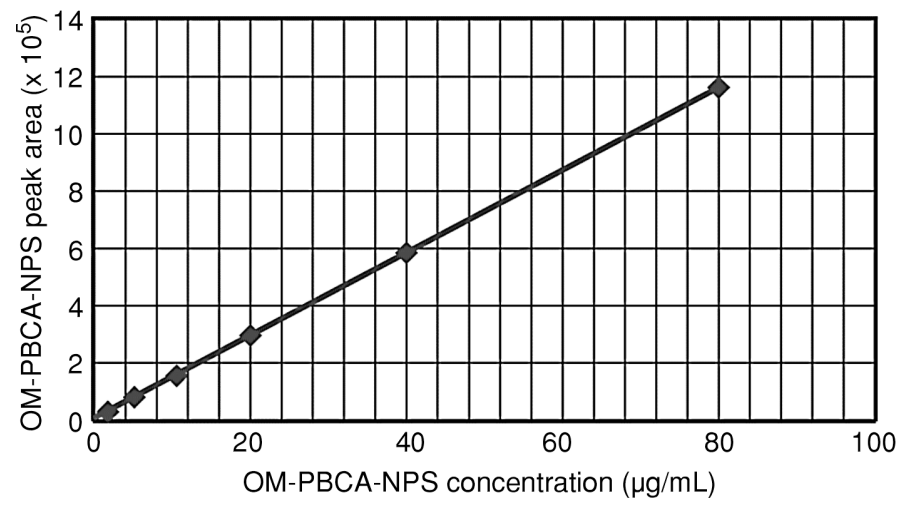

Figure 1. Standard curve of oxymatrine. Chromatographic conditions: the column was DiamonsilTM (diamond) C18 column $(250 \times 4.6$ $\mathrm{mm}, 5 \mu \mathrm{m})$, the mobile phase was acetonitrile- $0.02 \mathrm{M} \mathrm{KH}_{2} \mathrm{PO}_{4}$ buffer $(10: 90)$, with the flow rate as $1.0 \mathrm{~mL} / \mathrm{min}$, detection wavelength as $210 \mathrm{~nm}$, injection volume as $20 \mu \mathrm{L}$, and column temperature as room temperature. The regression equation: $\mathrm{A}=1448577.75 \mathrm{C}+1430.4$, $\mathrm{R}^{2}=0.9999$

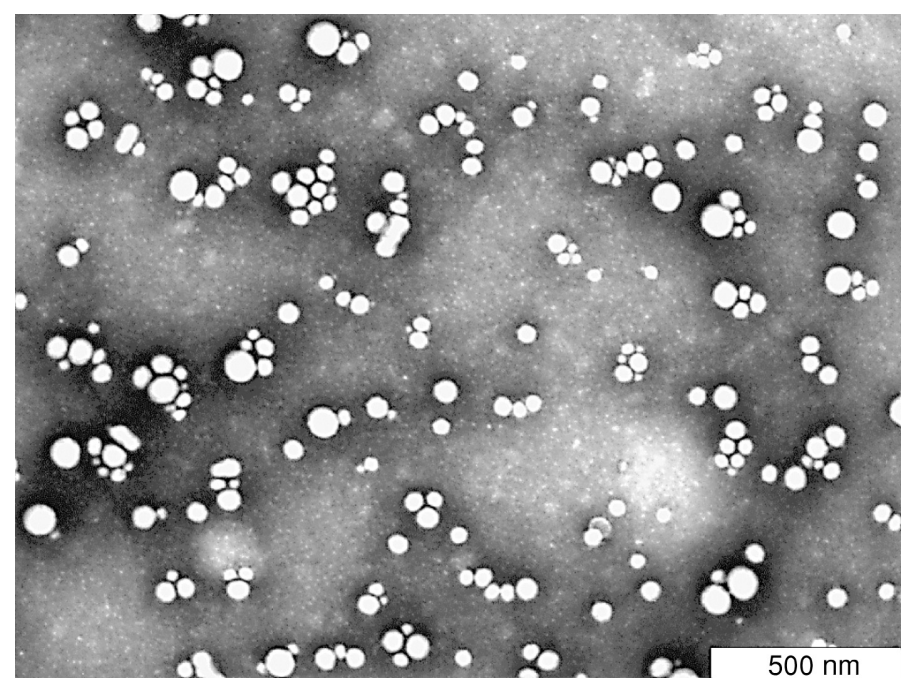

Figure 2. Negatively-stained TEM micrograph of OM-PBCA-NPs. 
t-test; the grade data were subjected to the non-parametric rank-sum test, with $\mathrm{p}<0.05$ being considered as statistical significance.

\section{RESULTS}

\section{Preparation of OM-PBCA-NP The standard regression line}

The peak area at different concentrations was shown in Table 1 and the linear regression was performed on that (Fig. 1). The regression equation was obtained: $\mathrm{A}=1448577.75 \mathrm{C}+1430.4, \mathrm{R}^{2}=0.9999$. The results showed that the peak areas and concentrations of oxymatrine showed a good linear relationship in the concentration range of $1 \mu \mathrm{g} / \mathrm{mL}$ to 80 $\mu \mathrm{g} / \mathrm{mL}$.

\section{Morphological observation}

Under the electron microscope (Fig. 2), it can be seen that the nanoparticles are evenly distributed, round and about $104 \mathrm{~nm}$ in size.

\section{Particle size and distribution}

The particle size of the nanoparticles was determined using one laser particle size analyzer $(104.1 \pm 29.11) \mathrm{nm}$ (Malvern), consistent with the results of TEM (Fig. 3).

\section{The encapsulation efficiency}

The encapsulation efficiency of OM-PBCANPs was $83 \%$.

\section{The drug loading}

According to the measured encapsulation efficiency and the administered amount of BCA, the drug loading of OM-PBCA-NPs was $16.6 \%$.
The determination of the content of oxymatrine in the nanoparticles required a suitable method to totally release oxymatrine from the nanoparticles; therefore, firstly, a suitable solvent or mixed solvents should be selected. However, considering the absence of the loss of drug amount added in the test, the nanoparticle colloidal solution containing the drug was subjected to ultrarapid centrifugation to separate the supernatant, and the concentration of the drug in the supernatant was determined by HPLC. The amount of the drug contained in the supernatant was deducted from the total drug amount, which was regarded as the drug amount encapsulated in the nanoparticles.

\section{Stability test}

The OM-PBCA-NPs colloidal solution was placed at room temperature $\left(20^{\circ} \mathrm{C}-30^{\circ} \mathrm{C}\right)$. After 3 months of storage, the appearance, particle size, encapsulation efficiency, and drug loading were all changed. The appearance gradually changed from uniform clear light white to pale yellow, and the particles precipitated. Under SEM, the degradation of OM-PBCA-NPs was obvious, which adhered to each other, the morphology changed, and the particle size increased, suggesting that the OM-PBCA-NPs colloidal solution was not very stable, so one more suitable dosage form should be selected to enhance the stability.

Protective effect of matrine nanoparticles on fatty liver injury in ob/ob mice

Effects of OM-PBCA-NPs on serum ALT and AST in ob/ob mice

The results showed (Table 2): compared with group MOD, the serum ALT and AST levels were significantly decreased in each group $(\mathrm{p}<0.05)$, and

Table 1. Results of the standard curve.

\begin{tabular}{|c|c|c|c|c|c|c|c|}
\hline Concentration $(\mu \mathrm{g} / \mathrm{mL})$ & 1 & 2 & 5 & 10 & 20 & 40 & 80 \\
\hline & 17929.8 & 27412.6 & 68871 & 147457.6 & 291512.8 & 589469.8 & 1156111.8 \\
Peak area $(\overline{\mathrm{X}} \pm \mathrm{SD})$ & \pm 14.9 & \pm 6.2 & \pm 3.7 & \pm 14.5 & \pm 10.1 & \pm 13.5 & \pm 9.2 \\
\hline
\end{tabular}



Figure 3. Particle size and Zeta potential value of OM-PBCA-NPs.Under TEM, the sizes of the nanoparticles exhibited uniform distribution, and the particles were round with the size of about $104 \mathrm{~nm}$. 
Table 2. Effects of OM-PBCA-NPs on ALT and AST in ob/ob mice.

\begin{tabular}{|c|c|c|c|}
\hline Group & Dose $(\mathrm{mg} / \mathrm{kg})$ & ALT & AST \\
\hline CON & - & $47.00 \pm 1.04$ & $122.37 \pm 1.76$ \\
\hline MOD & - & $134.76 \pm 0.99^{\#}$ & $140.16 \pm 1.02^{\#}$ \\
\hline OM & 30 & $66.88 \pm 1.26^{\Delta}$ & $125.32 \pm 1.42^{\Delta}$ \\
\hline OM & 205 & $59.33 \pm 1.03^{\Delta}$ & $154.33 \pm 1.54^{\Delta}$ \\
\hline SIL & 36 & $81.75 \pm 1.16^{\Delta}$ & $2.13^{\Delta}$ \\
\hline
\end{tabular}

Compare with group MOD, ${ }^{*} \mathrm{p}<0.05$; compare with group $\mathrm{CON},{ }^{\Delta} \mathrm{p}<0.05,{ }^{\Delta 4} \mathrm{p}<0.01$.

Table 3. Effects of OM-PBCA-NPs on blood lipids in ob/ob mice.

\begin{tabular}{|c|c|c|c|c|c|}
\hline Group & Dose $(\mathrm{mg} / \mathrm{kg})$ & TC & TG & LDL & HLDL \\
\hline CON & - & $2.69 \pm 0.98$ & $1.12 \pm 1.07$ & $0.54 \pm 1.35$ & $2.43 \pm 1.81$ \\
MOD & - & $4.04 \pm 2.20 ?$ & $1.50 \pm 1.02^{\#}$ & $1.48 \pm 1.48^{\#}$ & $3.73 \pm 1.18^{\#}$ \\
OMT & 30 & $3.32 \pm 1.58^{\Delta}$ & $0.93 \pm 2.13^{\Delta}$ & $0.99 \pm 2.44^{\Delta}$ & $3.06 \pm 2.02^{\Delta}$ \\
OM & 205 & $2.55 \pm 1.03^{\Delta}$ & $0.89 \pm 1.42^{\Delta}$ & $0.97 \pm 2.33^{\Delta}$ & $2.16 \pm 1.91^{\Delta}$ \\
SIL & 36 & $3.95 \pm 1.16$ & $1.37 \pm 301$ & $1.06 \pm 1.47$ & $3.55 \pm 1.75$ \\
\hline
\end{tabular}

Notes: The CON, MOD, OMT, OM, and SIL respectively represent the normal group, model group, OMT group, OM-PBCA-NP group, and silymarin group. Compared with group $\mathrm{CON},{ }^{*} \mathrm{p}<0.05$; compared with group MOD, ${ }^{\Delta} \mathrm{p}<0.05$

group OM $>$ group OMT $(\mathrm{p}<0.05)>$ group SIL $(\mathrm{p}<0.05)$.

\section{Effects of OM-PBCA-NPs on blood lipid content in ob/ob mice}

The results showed (Table 3): Compared with group MOD, the blood lipid level of the mice in group OM and group OMT were significantly decreased $(\mathrm{p}<0.05)$; there was no significant change in group SIL.

OM-PBCA-NPs can reduce the blood lipid and serum ALT/AST levels in ob/ob mice, indicating that oxymatrine nanoparticles have certain hepatoprotective effects and are superior to traditional oxymatrine granules.

\section{DISCUSSION}

The incidence of fatty liver injury has increased rapidly in recent years, which is a serious threat to human health and has been recognized as a common cause of occult cirrhosis (18-20). Studies have reported that oxymatrine can protect the liver cell membrane, inhibit the release of fibrotic factors, regulate the function of fat storage cells, and exert anti-fibrosis and anticirrhosis effects in multiple links $(4,5)$. At present, applications of oxymatrine injection, capsules, or tablets, have been clinically used. However, common preparations require multi-administration, together with large dosages, great blood concentration fluctua- tion in patients, and large toxic side effects (such as nausea or vomiting), which brings certain difficulties and problems to clinical applications of oxymatrine.

According to the principle of the polymerization reaction, there are two main preparation methods of n-butyl polycyanoacrylate nanoparticles, namely the emulsion polymerization method and the interfacial polymerization method (21). The Interfacial polymerization method is suitable for the preparation of fat-soluble drug-loaded nanoparticles (22). The emulsion polymerization method can be further divided into the emulsion polymerization method (one-step method) and the blank adsorption method (two-step method) according to the drug addition order. The one-step method is to dissolve the drug and dextran (Dextran) or non-ionic surfactants, such as Pluronic F68, Tween, Brij 96, etc. in an acidic aqueous solution, together with slow addition of BCA monomer under continuous stirring for a sufficient time, so that the BCA monomer can be automatically polymerized to form nanoparticles under the catalysis of water or dextran. This method is simple and convenient and is suitable for the preparation of water-soluble drug-loaded nanoparticles (23). In the one-step method, the binding modes between the drug and the carrier include encapsulation, entrainment, or adsorption; the drug in the twostep method is mainly adsorbed on the surface of nanoparticles so that the encapsulation efficiency of the one-step method is higher. 
Particle size is an important quality indicator for nanoparticle delivery systems. For passively targeted preparations, the size of the particle s directly affects the distribution of the drug in vivo. Nanoparticles with different sizes have different in vivo distribution characteristics (24). From the perspective of medication safety, the smallest capillary diameter of the human body is about $4 \mu \mathrm{m}$, so the size of the intravenously injected nanoparticles should be less than this limit. From the perspective of in vivo targeting, particles with the size being less than $50 \mathrm{~nm}$ can penetrate the liver endothelium or reach the spleen, bone marrow, or tumor cells through the lymphatic system; particles with the size being more than $100 \mathrm{~nm}-2 \mu \mathrm{m}$ can be phagocytized by the reticuloendothelial system (RES), those with the size within 2-7 $\mu \mathrm{m}$ ingested by the capillary vascular network are accumulated in the lungs, spleen, and liver, and those with the size within 7-12 $\mu \mathrm{m}$ are more mechanically blocked by the lungs (25). The OM-PBCA-NPs prepared in this study for liver targeting has the particle size of $(104.1 \pm 29.11) \mathrm{nm}$ and narrow distribution, which met the requirements of liver targeting particle size. The particle size measurement by laser scattering particle size analyzer was simple, quick, and easy: putting the dispersion medium and the test sample, starting the ultrasonic generator to fully disperse the sample, and then starting the circulation pump. The actual test process was only a few seconds. The method was quick and the cost was low.

In this study, OM-PBCA-NPs was prepared by emulsion polymerization, which was a targeted drug delivery system, and can reduce the systemic distribution and dosage, thus reducing the medication times, improving the therapeutic indexes of the drug, reducing adverse reactions, and exhibiting very important application prospects.

$\mathrm{Ob} / \mathrm{ob}$ mice are leptin-deficient mice. $\mathrm{Ob}$ is a leptin-encoding gene, which exon 2 and 3 of the ob gene are knocked out to obtain ob/ob leptin-deficient mice. Its genetic background is $\mathrm{C} 57 \mathrm{BL} / 6 \mathrm{~J}$ mice. Therefore, $\mathrm{C} 57 \mathrm{BL} / 6 \mathrm{~J}$ was chosen as the normal group (26). Ob/ob mice were used as model animals to verify the hepatoprotective effects of OMPBCA-NPs. The results demonstrate that OMPBCA-NPs can reduce blood lipid and serum ALT/AST levels in ob/ob mice. The effects are better than traditional oxymatrine granules, which provides strong support for the further development and utilization of n-butyl polycyanoacrylate nanoparticles, and its pharmacological action mode needs further studies.
N-butyl polycyanoacrylate nanoparticles are dispersed in a medium in the form of colloidal particles and belong to a thermodynamically unstable system (27). Stability tests have shown that choosing a more suitable dosage form to enhance stability is the top priority for the next steps. Referring to relevant studies, it can be known that preparing the nanocolloid solution into lyophilized powder can be beneficial to long-term storage of such products (28, 29), which can ensure good dispersibility and physicochemical stability, facilitate subsequent intravenous administration into test animals, and provide favorable conditions for further exploring related targeted drug delivery system.

\section{Acknowledgments}

Scientific research project of Ningxia Medical University (XM2018010); Innovation and entrepreneurship project for first-class discipline college students of College of traditional Chinese medicine of Ningxia Medical University (NXYLXK2017A06).

\section{Conflicts of interest}

The authors declare no conflict of interest.

\section{REFERENCES}

1. Shi H.J., Zhou H., Ma A.L., Wang L., Gao Q., et al.: Br. J. Dermatol. 181, 1028 (2019).

2. Zuo M.L., Wang A.P., Tian Y., Mao L., Song G.L., et al.: J. Cell. Biochem. 120, 16185 (2019).

3. Liu Y., Xu Y., Ji W., Li X., Sun B., et al.: Tumour Biol. 35, 5111 (2014).

4. Wang Y.P., Zhao W., Xue R., Zhou Z.X., Liu F., et al.: Antiviral Res. 89, 227 (2011).

5. Lin Y.Y., Chou C.F., Giovarelli M., Briata P., Gherzi R., et al.: Mol. Cell. Biol. 34, 2339 (2014).

6. Li L., Liu Q., Fan L., Xiao W., Zhao L., et al.: Oncotarget 8, 12792 (2017).

7. Pei X., Wang W., Miao N., Xu M., Zhang C., et al.: Toxicol. Pharmacol. 37, 423 (2014).

8. Liu M., Jin S., Yan H., Du S.: Eur. J. Pharm. Sci. 104, 212 (2017).

9. Lu H., Zhang L., Gu L.L., Hou B.Y., Du G.H.: Basic Clin. Pharmacol. Toxicol. 119, 405 (2016).

10. Shi H.J., Song H.B., Wang L., Xiao S.X., Bo K.P., et al.: Biomed. Pharmacother. 97, 19 (2018). 
11. Zhou F., Cai X.J., Ni J.J., Cao J.W., Xu Y.Y.: Zhejiang 25, 7 (2015).

12. Peng X.S., Li B.H., Hu M., Ling Y., Tian Y., et al.: J. Anal. Methods Chem. 2014, 4 pages (2014).

13. Liu Q.A., Li Q.A., Huang Y.B., Cai T.C., Cai Y.D.: J. Biomater. Tissue Eng. 7, 582 (2017).

14. Rajpoot K.: Curr. Pharm. des. 37, 3943 (2019).

15. Xu C.Y., Yan Y.F., Tan J.C., Yang D.H., Jia X.J., et al.: Adv. Funct. Mater. 29, 1808146 (2019).

16. He W., Hong Y.: China Medical Herald 14, 13 (2016).

17. Li L.J.: J. Changsha Medical University 11, 3 (2013).

18. Allard J., Le Guillou D., Begriche K., Fromenty B.: Adv. Pharmacol. 85, 75 (2019).

19. Liangpunsakul S., Chalasani N.: Am. J. Physiol. Gastrointest. Liver Physiol. 316, G75 (2019).

20. Li S., Fujino M., Takahara T., Li X.K.: Med. Mol. Morphol. 52, 61 (2019).
21. Antonietti M., Landfester K.: Prog. Polym. Sci. 27, 689 (2002).

22. Jönsson J.B., Müllner M., Piculell L., Karlsson O.J.: Macromolecules. 46, 9104 (2013).

23. Cho Y.S., Ji S., Kim Y.S.: J. Nanosci. Nanotechnol. 19, 6398 (2019).

24. Toome K., Willmore A.A., Paiste P., Tobi A., Sugahara K.N., et al.: Nanoscale 9, 10094 (2017).

25. Yuan Q., Wang Y., Song R., Hou X., Yu K., et al.: Front. Pharmacol. 10, 225 (2019).

26. Cui H.H., Li J.B., Chai S.Q., Ming Y., Wang D.J., et al.: J. Chongqing Medical University 39, 7 (2014).

27. Hertel P., Thirring W.: Quanten und Felder, pp. 309-324, Springer-Verlag Berlin Heidelberg GmbH (1971).

28. Lloyd I.K., Blachere J.R., Beyer L.A., Kalnas C.E., Mairet P.A.: Ultramicroscopy 29, 153 (1989).

29. Rao S., Song Y., Peddie F., Evans A.M.: Int. J. Nanomedicine 6, 1245 (2011).

(C) 2020 by Polish Pharmaceutical Society. This is an access article under the CC BY NC license 